\title{
REVISÃO SISTEMÁTICA DE REVISÕES DA LITERATURA SOBRE A SÍNDROME DE BURNOUT EM DOCENTES DO ENSINO SUPERIOR NO BRASIL
}

\author{
Dartel Ferrari de Lima (D1, Lohran Anguera Lima iD2 \\ Adelar Aparecido Sampaio ${ }^{\text {iD } 3}$ e Claus Dieter Stobaus iD4
}

\section{Resumo}

Este estudo analisa como os especialistas operacionalizam revisões publicadas em periódicos focando a síndrome de burnout. A revisão sistemática das revisões, realizada com recorte temporal de janeiro de 2010 a fevereiro de 2020, mostrou estudos quantitativamente limitados de revisões da literatura sobre o tema em foco. A maior parte das revisões encontradas são voltadas a investigar professores da área da saúde. Os estudos geralmente apresentam assimetrias nos aspectos metodológicos, limitando sobremaneira a comparação direta dos resultados. Entretanto, evidenciou-se a necessidade urgente de estudos de revisão da literatura adotarem protocolos sistematizados para nortear as futuras revisões sistemáticas para oferecer critérios mais transparentes e confiáveis, minimizando suposições implícitas e vieses de acometimento da síndrome de burnout em professores universitários.

Palavras-chave: Bem-estar docente; Saúde docente; Estresse ocupacional; Síndrome de burnout; Esgotamento.

\section{SYSTEMATIC REVIEW OF LITERATURE REVIEWS ON BURNOUT SYNDROME IN HIGHER EDUCATION TEACHERS IN BRAZIL}

\begin{abstract}
This study examines how experts operationalize reviews published in journals focusing on burnout syndrome. The systematic review of reviews, conducted from January 2010 to February 2020, showed quantitatively limited studies of literature reviews on the topic in focus. Most of the reviews found are focused on investigating health professors. The studies generally present asymmetries in methodological aspects, greatly limiting the direct comparison of results.
\end{abstract}

${ }^{1}$ Doutorado em Ciências pela Faculdade de Medicina da Universidade de São Paulo, São Paulo, São Paulo, Brasil. Professor Permanente do Programa de Pós-Graduação em Educação em Ciências e Educação Matemática pela Universidade Estadual do Oeste do Paraná, Marechal Cândido Rondon, Paraná, Brasil.

${ }^{2}$ Especialização em Ortopedia e Traumatologia. Residente do Departamento de Ortopedia e Traumatologia da Santa Casa de Misericórdia de São Paulo, São Paulo, Brasil.

${ }^{3}$ Doutorado em Educação pela Pontifícia Universidade Católica do Rio Grande do Sul, Porto Alegre, Rio Grande do Sul, Brasil. Professor da Universidade Federal de Mato Grosso do Sul Campus do Pantanal (UFMS/CPAN).

${ }^{4}$ Doutorado em Ciências Humanas - Educação pela Universidade Federal do Rio Grande do Sul (UFRGS), Pós-doutorado em Psicologia da Educação pela UAM, Espanha. 
However, it was evident the urgent need for literature review studies to adopt systematized protocols to guide future systematic reviews to offer more transparent and reliable criteria, minimizing implicit assumptions and biases of burnout syndrome in college professors.

Keywords: Teaching well-being; Teaching health; Occupational stress; Burnout syndrome; Exhaustion.

\section{Introdução}

Tradicionalmente, empreender uma revisão da literatura é concebido como um método de estudo secundário e, que têm nos estudos primários, a sua fonte de dados. Isso possibilita aos revisores, mapear o território intelectual existente sobre determinado assunto. Assim, a revisão bibliográfica é considerada um exercício crucial para expor os limites da pesquisa com base no uso de resultados de estudos anteriores. Ressalta-se, ainda, que a revisão da literatura permite filtrar os diferentes estudos conforme o seu grau de contribuição, permitindo ao revisor, apresentar uma visão ampliada do conhecimento do estado da arte.

É importante enfatizar que existem diferentes categorias de revisão da literatura, cada uma assumindo diferentes expressões relacionadas com o seu grau de sistematização e a função a que se destinam. A revisão sistemática é considerada o estudo observacional retrospectivo (CAMPBELL et al., 2020), mais utilizado nas pesquisas na área da Saúde (SOUZA et al., 2018). Se estrutura como um protocolo de métodos objetivos que fornece a descrição detalhada de cada passo da investigação prospectiva (WORMALD; EVANS, 2017). Desse modo, pesquisadores de diferentes áreas do conhecimento têm despendido esforços para expor o que se sabe sobre as alterações do bem-estar de professores universitários e possível instalação de burnout decorrente de novas relações de trabalho. O refinamento do debate sobre essa questão merece exame detalhado.

Assim, ultrapassada a necessidade de apresentar a revisão da literatura como instrumento de pesquisa, faz-se necessário contextualizar a revisão da literatura e focar na interação das relações sociais contemporâneas com as relações de trabalho, potencializando níveis de insatisfação, sofrimento, sentimento de felicidade e a satisfação do trabalhador docente do Ensino Superior.

Mais recentemente, há muitas tentativas de lançar luz sobre os fenômenos que afetam o bem-estar dos professores brasileiros. Pesquisadores observaram um foco crescente de manifestações de mal-estar dos docentes, com repercussão negativa na sua saúde e no seu rendimento, inicialmente atribuídas às condições de trabalho (SAMPAIO; STOBÄUS; BAEZ, 2017; SAMPAIO et al., 2021). 
A manifestação de mal-estar reportada nesses trabalhos apresenta como característica um estado de exaustão emocional, podendo culminar em burnout, resultante das relações sociais complexas, das projeções pessoais e da cronificação de um processo de estresse (FERREIRA; BORGES, 2010. Esse quadro sintomático é semelhante ao descrito no ano de 1980 , pelo psicólogo Herbert J. Freudenberger (1926-1999), na publicação Burn Out: The High Cost of High Achievement. What it is and how to survive it? (original em inglês), levando ao uso atual da expressão 'síndrome de burnout' como caracterização do fenômeno.

A síndrome de burnout, psicopatologia laboral de grande risco na docência, tem sido definida como um fenômeno psicossocial que emerge como uma resposta crônica dos estressores interpessoais ocorridos na situação de trabalho. Constitui-se de três dimensões relacionadas, mas independentes: 1) exaustão emocional caracterizada pela falta ou carência de energia, entusiasmo e sentimento de esgotamento de recursos; 2) despersonalização, situação em que o profissional passa a tratar os clientes, colegas e a organização como objetos; 3) baixa realização pessoal no trabalho, definida como a tendência do trabalhador em se autoavaliar de forma negativa (FERREIRA et al., 2015).

Na categoria profissional docente, a síndrome de burnout age como um ciclo degenerativo de sua eficácia laboral, configurada como um conjunto de consequências negativas que afetam o professor a partir de uma ação combinada de condições psicológicas e sociais em docentes, não devendo ser confundido com estresse (CODO, 1999).

A síndrome de burnout não recebe a mesma atenção dispensada a outros fatores de proteção ao trabalho e ao trabalhador (SZELIGA-KOWALCZYK, 2015). Talvez isso decorra da complexidade que é a interação de fatores psicossociais na docência, dificultando determinar com exatidão a dimensão do fenômeno. Esse padrão de argumentação ainda demanda explicações, a fim de ampliar a compreensão desse dimensionamento.

Embora várias revisões da literatura tenham sido realizadas nesse sentido, abordagens e princípios básicos subjacentes nessas avaliações não são bem conhecidos, e parte dessas publicações mostra um grau de inconsistência de informações. O desacordo com as orientações metodológicas (qualidade) e o viés de publicação - tendência maior de publicações para estudos com evidências positivas, quando comparadas aos estudos com evidências negativas - podem enviesar os resultados disponíveis para confronto (PAGE et al., 2016).

Conhecer a operacionalização das revisões da literatura focadas na saúde, bem-estar e rendimento docente no ensino superior pode contribuir para aperfeiçoar a execução de futuras revisões, melhorando a qualidade e a confiança sobre estas informações. Com base nessa premissa, este estudo teve a seguinte questão norteadora: Qual o padrão de qualidade da produção científica nacional acerca das revisões da literatura para o tema 'síndrome de burnout' em docentes universitários? 


\section{Material e métodos}

Este estudo se estruturou como uma revisão sistemática de revisões de literatura sobre a síndrome de burnout afetando professores universitários, realizada para identificar como os estudiosos nesse campo conduziram e relataram as revisões da literatura apresentadas nos periódicos da área. Esta revisão foi registrada no International Prospective Register of Systematic Reviews - PROSPERO, sob o número 175454, do Instituto Nacional de Pesquisa em Saúde, com sede em York, Reino Unido (National Institute for Health Research - NIHR), em 19/03/2020, um banco de dados internacional de revisões sistemáticas ${ }^{1}$, para evitar duplicação de estudo e reduzir a oportunidade de viés de relatórios. Foi conduzida seguindo a lista de verificação dos itens de relatório preferenciais para análises sistemáticas e metanálises The PRISMA Statement (Preferred Reporting Items for Systematic Review and Meta-analyses) e alinhada às recomendações da Cochrane Collaboration, organização internacional não governamental com sede em Oxford, Reino Unido, com missão de preparar, manter e assegurar 0 acesso às revisões sistemáticas de intervenções em Saúde, e do The PRISMA Statement, conjunto mínimo de itens com base em evidências para relatórios em revisões sistemáticas e metanálises.

\subsection{Critérios de elegibilidade}

Este estudo incluiu revisões da literatura com a temática síndrome de burnout em professores do ensino superior, indexadas nas bases de dados selecionadas previamente, com resumos e texto disponíveis na íntegra com documentos disponíveis por meio digital, publicados no Brasil em língua portuguesa (Brasil), inglesa e espanhola, no período de janeiro de 2010 a fevereiro de 2020. Foram excluídos estudos de revisão que envolveram na amostra docentes de outros níveis de ensino (não superior) ou de outra nacionalidade (não brasileira), com foco na depressão ou no estresse dos professores e registros apresentados em formato de resumos expandidos.

\subsection{Estratégia de busca}

As buscas foram realizadas nas bases de dados eletrônicas MEDLINE (Medical Literature Analysis and Retrieval System on-line) via PubMed, Portal de Periódicos da Coordenação de Aperfeiçoamento de Pessoal de Nível Superior (CAPES) vinculada ao Ministério da Educação (MEC), Biblioteca Eletrônica Científica Online (Scielo - Scientific Electronic Library Online) e Biblioteca Virtual em Saúde (BVS). A estratégia de busca incluiu os descritores propostos pelos Descritores em Ciências da Saúde (DeSC) da Biblioteca Virtual em Saúde - que é a tradução do Medical Subject Headings (MeSH on Demand) - referente a desfechos esperados (burnout), a população envolvida (professor/es universitário/s) e o tipo de estudo (revisão sistemática) com uso do operador booleano $A N D$, refinado por data de publicação para o período de 2010 a 2020. Para o último ano do período, exceto para o Portal de Periódicos CAPES/MEC, foram consideradas as revisões publicadas até o mês de fevereiro. $O$ Portal de

${ }^{5}$ Disponível em: https://www.crd.york.ac.uk/prospero/\#myprospero 
Periódicos Capes/MEC não disponibiliza resultados de ciclos anuais em andamento. A estratégia de busca utilizou os descritores para busca em três idiomas, proporcionando um meio consistente e único para a recuperação de informação. A última coleta foi realizada em 20 de março de 2020.

\subsection{Seleção dos estudos e extração dos dados}

Inicialmente foram selecionados textos completos, identificados pela estratégia de busca, sendo avaliados de forma independente por dois revisores (SCM/SP e Unioeste/PR). Um selecionou os registros incluídos e o outro verificou os registros excluídos. Os desacordos foram resolvidos por consenso. Na falta de consenso, foram consideradas a decisão de um terceiro revisor. Posteriormente, todos os relatórios incluídos foram revisados cuidadosamente, para extrair e codificar as informações. Incluímos todos os artigos de revisão que, em certa medida, mostraram que os métodos usados para identificar e selecionar a literatura eram explícitos. Os periódicos foram classificados de ' $A$ ' a ' $C$ ', seguindo a classificação de sistema Qualis da Capes para o quadriênio 20132016, obtidos pelo acesso à Plataforma Sucupira.

\section{Resultados e discussões}

\subsection{Processo de seleção dos estudos}

As buscas realizadas nas bases de dados selecionadas identificaram preliminarmente 246 registros, dos quais, após a avaliação do título e resumo, foram excluídos 215 estudos (que não atenderam os critérios de elegibilidade, ou por desvio de tema ou, de população alvo). Diante dos 31 registros resultantes da análise preliminar, a inspeção do texto completo identificou e excluiu outros 19 registros com desvios de meta. Na etapa do processo de elegibilidade restaram 12 registros, dos quais 6 constavam em mais de uma base de dados e foram excluídos pelo critério de duplicidade. Dessa forma, foram separados 6 registros, sendo todos considerados elegíveis e potencialmente relevantes para a revisão. O reduzido número de trabalhos analisados deve ser ponderado na indicação de certeza dos resultados, evidenciado na Figura 1. 


\section{Figura 1. Diagrama do fluxo PRISMA da estratégia de busca adotada.}
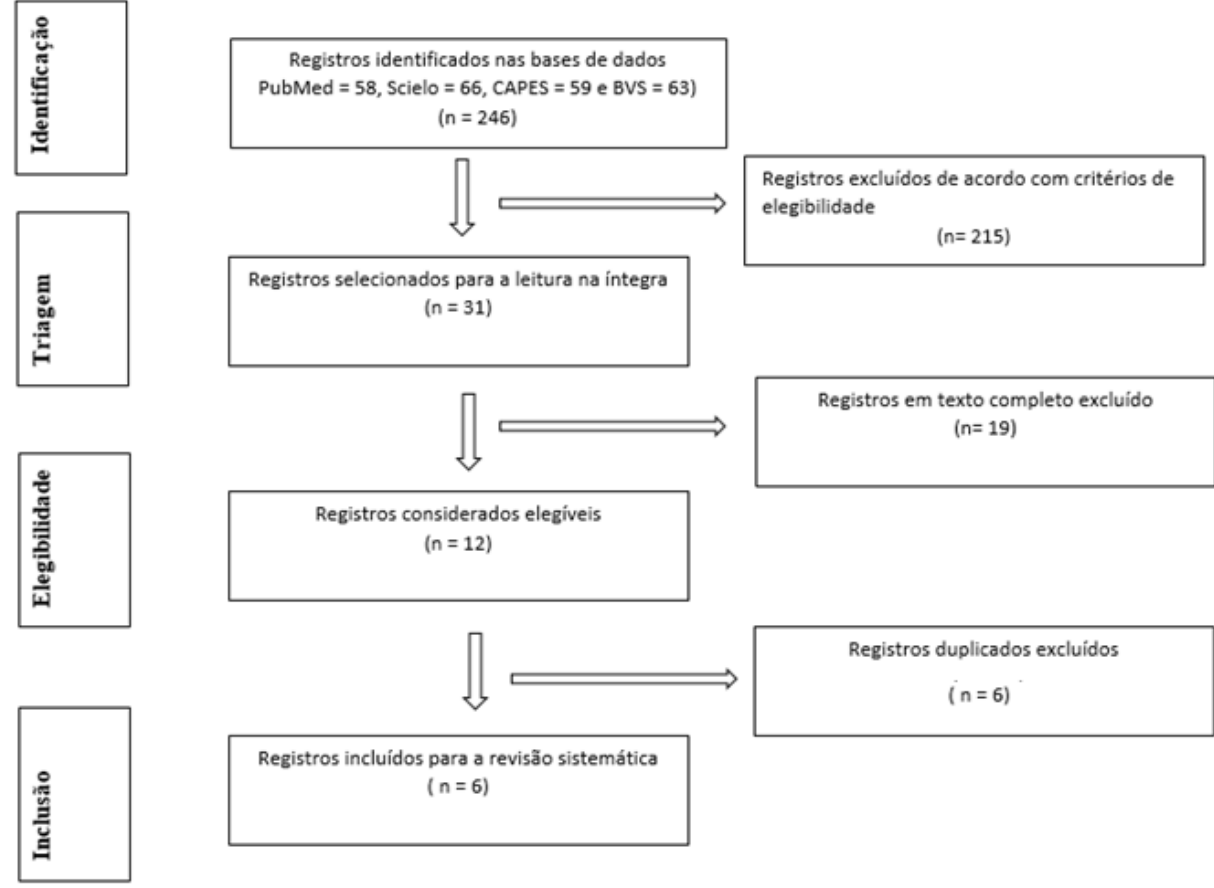

Fonte: Autores (2021).

\subsection{Características dos estudos selecionados}

Para a apresentação dos registros selecionados foram identificados os registros elegíveis quanto à autoria, classificação do periódico e o ano da publicação. Os resultados mostraram que o volume de realização sistemática de revisões ao longo do tempo se concentrou no período de 2015 e 2016 (60\%). Não foram encontrados registros no Sistema Qualis da Capes para o quadriênio 2013-2016, de artigos em periódicos com estrato ' $A$ '. Os periódicos B1 e B2 abrigaram $33 \%$ dos registros, enquanto os demais completaram a distribuição (PEREIRA et al., 2018; FREITAS; SENRA, 2015; MARTINS et al., 2016; LEITE; NOGUERIA, 2017; FERREIRA; BORGE, 2010) (Anexo 1).

A síntese das revisões elencadas foi norteada por adaptação do sistema PRISMA. O sistema original consiste em um checklist com 27 itens com o objetivo de qualificar o relato de revisões sistemáticas e metanálises. Apesar do foco originário, idealizado para qualificar ensaios clínicos randomizados, o PRISMA também tem sido utilizado como instrumento orientador de análises críticas de revisões sistemáticas publicadas.

Os títulos das revisões elencadas neste estudo anunciaram a categoria de estudo e a que se propunha; foram artigos publicados em diferentes periódicos, de diferentes instituições. Nas revisões, a quantidade de estudos encontrados na primeira inspeção foi heterogênea, variando de 7 a 532 (média 163). A mesma variação heterogênea ocorreu para os estudos eleitos, variando de 3 a 20 estudos (média 9). Os demais indicadores do PRISMA podem ser conferidos na Tabela 1. 
Tabela 1. Apresentação do resumo dos registros elegíveis de revisões da literatura buscadas em banco de dados eletrônicos para os descritores 'síndrome de burnout' AND 'professor(es) universitário(s)', segundo o com a lista de verificação do PRISMA.

Tabela 1 - Resumos com os descritores 'síndrome de burnout' AND 'professor(es) universitário(s)'.

\begin{tabular}{|c|c|c|c|c|c|c|}
\hline REGISTROS & 1 & 2 & 3 & 4 & 5 & 6 \\
\hline Revisão sistemática & $\mathrm{X}$ & & & & & \\
\hline Revisão narrativa & & & & & & $\mathrm{X}$ \\
\hline Revisão integrativa & & & $\mathrm{X}$ & $\mathrm{X}$ & & \\
\hline Revisão crítica/bibliométrica & & $\mathrm{X}$ & & & $\mathrm{X}$ & \\
\hline Título $^{1}$ & Sim & Sim & Sim & Sim & Sim & Sim \\
\hline Resumo estruturado ${ }^{2}$ & Sim & Não & Sim & Sim & Não & Não \\
\hline Problematização ${ }^{3}$ & Sim & Não & Sim & Sim & Sim & Não \\
\hline Objetivo explícito ${ }^{4}$ & Sim & Não & Sim & Sim & Sim & Não \\
\hline $\begin{array}{l}\text { Referência para conduzir a } \\
\text { revisão }{ }^{4}\end{array}$ & Sim & Não & Sim & Sim & Sim & Não \\
\hline Ano da publicações ${ }^{4}$ & 2018 & 2015 & 2016 & 2017 & 2010 & 2020 \\
\hline Data da pesquisa ${ }^{4}$ & $06 / 2018$ & NI & $07 / 2015$ & NI & $04 / 2010$ & NI \\
\hline Tamanho da equipe & 4 & 2 & 5 & 2 & 2 & 2 \\
\hline Dois revisores independentes 4 & Sim & NI & Sim & NI & NI & NI \\
\hline$N^{0}$ de estudos encontrados ${ }^{4}$ & 7 & 300 & 532 & 81 & 60 & NI \\
\hline $\mathrm{N}^{0}$ de duplicatas ${ }^{4}$ & NI & NI & 32 & NI & NI & NI \\
\hline $\mathrm{N}^{0}$ de trabalhos excluídos ${ }^{4}$ & 4 & 282 & 529 & 61 & 48 & NI \\
\hline $\mathrm{N}^{\circ}$ de registro examinados & 3 & 18 & 3 & 20 & 12 & NI \\
\hline $\begin{array}{l}\text { Tamanho da amostra dos } \\
\text { estudos }\end{array}$ & Não & Não & Não & Sim & Sim & Sim \\
\hline Lista de artigos incluídos & Sim & Sim & Sim & Sim & Sim & Não \\
\hline Sínteses dos resultados & Sim & Sim & Sim & Sim & Sim & Não \\
\hline $\begin{array}{l}\text { Discussão e conclusão } \\
\text { agrupadas }\end{array}$ & Sim & Sim & Sim & Sim & Sim & Não \\
\hline Resumo das evidências & Sim & Sim & Sim & Sim & Sim & Não \\
\hline Limitações do estudo & Não & Não & Sim & Não & Não & Não \\
\hline Conclusões & Sim & Sim & Sim & Sim & Sim & Não \\
\hline $\begin{array}{l}\text { Sugestões para futuras } \\
\text { pesquisas }\end{array}$ & Sim & Sim & Sim & Sim & Não & Não \\
\hline Financiamento & NI & NI & NI & NI & NI & NI \\
\hline Idiomas & $\mathrm{P}, \mathrm{I}, \mathrm{E}$ & $P$ & $\mathrm{P}, \mathrm{I}, \mathrm{E}$ & $\mathrm{P} ; \mathrm{I}$ & $\mathrm{P}, \mathrm{I}, \mathrm{E}$ & NI \\
\hline Qualificação dos autores & Sim & Sim & Sim & Sim & Sim & NI \\
\hline Fontes de pesquisa & $a ; b ; c ;$ & d & $a ; b ; e$ & a; c; d; & a; c & NI \\
\hline
\end{tabular}

Fonte: Adaptado pelos autores do PRISMA (MOHER et al., 2012).

${ }^{1}$ Informa o tipo da revisão; ${ }^{2}$ indica as etapas do trabalho; ${ }^{3}$ coerência da proposta; ${ }^{4}$ verificado a presença ou ausência; NI - não informado; P - português; I - inglês; E - espanhol; a) LILACS; b) PubMed; c) Scielo; d) Goolge Acadêmico; e) CINAHL; f) Periódicos Capes. PRISMA - Itens de relatório elegidos para revisões sistemáticas e metanálises (Preferred Reporting Items for Systematic Reviews and Meta-Analyses) 
Inicialmente, destaca-se a relevância de estudos sobre burnout em professores universitários, ainda escassa, com revisões da literatura publicadas que investigam sobre essas temáticas. Assim, pode-se explicar talvez porque a busca dessas revisões resultou em apenas seis estudos. Embora sejam poucas revisões, todas evidenciaram um viés de publicação seletiva de desfecho, com tendência a menor citação de estudos com resultados negativos. Essa tendência do viés de publicação pode levar, geralmente, na direção da superestimação da associação, ou seja, que apresentam associação entre exposição e desfecho. A discussão foi processada considerando quatro tópicos de interesse: título e resumo; introdução, métodos, e resultados e conclusão.

\subsection{Título e resumo das revisões elencadas}

A busca de estudos normalmente gera um número muito maior de artigos do que os que realmente serão elegíveis pelos critérios estabelecidos. Quando se trata de triagem de estudos para a inclusão em uma revisão da literatura, a leitura rápida do título e resumo permite descartar grande número de referências sem critérios de elegibilidade estabelecidos nesta revisão, que encontrou 246 registros (Figura 1), permanecendo apenas seis deles. A maior parte das exclusões foi realizada já pelo título e/ou resumo.

Os moldes exigidos para a publicação de revisão da revisão Cochrane Library (HIGGINS; GREEN, 2013) estabelece que o título deve ser apresentado em linguagem simples. A linguagem simples é uma técnica de comunicação com objetivo de apresentar um texto que seja entendido logo na primeira leitura, que permita localizar com rapidez a informação e entendê-la. De modo adicional, a declaração Strengthening the Reporting of Observational Studies in Epidemiology (STROBE) recomenda que se indique o desenho do estudo no título (VON ELM et al., 2007).

Habitualmente, após o uso dos descritores de busca, o título se torna a primeira orientação para compor a escolha de estudos para a revisão da literatura. A qualidade do título é o 'cartão de visita' do estudo e o erro mais comum, seja por descuido de revisão, seja por falha editorial, seja por qualquer outro motivo que o justifique, põe em risco a escolha por suspeita de qualidade. Foi observado nesta revisão somente uma falha em um dos títulos ao descrever a síndrome de esgotamento profissional como 'síndrome de 'Bornout' ao invés de 'burnout'. Neste caso específico, o registro mencionado foi mantido como originalmente escrito para ser exposto a outras análises de interesse central deste estudo.

Os títulos de todos os artigos elencados neste estudo continham a palavra 'revisão', se alinhando às recomendações STROBE. Algumas das revisões identificaram seu relatório como revisão integrativa ou sistemática. Uma revisão intitulou tratar-se de revisão bibliométrica, no entanto, na análise integral do texto, não foi observado tratamento metodológico para tal (ARAÚjO, 2006). A revisão não dispensou nenhuma medida quantitativa ou análise estatística para tratar os resultados. Pode-se, nesse caso, concluir por um título 'equivocado'. 
Nem sempre é possível descartar o estudo após leitura do título. Isso se deve à rotulação (in)adequada do desenho de estudo e, por isso, frequentemente é necessária a leitura do resumo para verificação do desenho de estudo de fato empregado.

Os resumos constituem, habitualmente, a segunda fonte inspeção de um estudo selecionável. Nem todas as bases de dados eletrônicas são de livre acesso, limitando este ao título e ao resumo da publicação. Neste estudo, 50\% das revisões foram estruturados e abordaram os métodos adequadamente. $A$ falta de estruturação pode ser parcial, devido ao formato prescrito pelos periódicos. A totalidade das revisões descreveram problematização e justificativa do estudo, detalhando as razões para executar a pesquisa, mas não apresentaram referencial teórico de embasamento da revisão.

\subsection{Introdução das revisões elencadas}

$\mathrm{Na}$ seção Introdução, de modo geral, esperava-se encontrar a sustentação do estudo com base em referências apropriadas que amparassem o trabalho. Esse fundamento foi presente em um único trabalho, curiosamente, a partir do grupo de autores com maior graduação, quando comparado aos pares das outras revisões elencadas.

Com foco na problematização, alguns 'desencontros' na Introdução foram percebidos. Um dos trabalhos contextualizou o impacto negativo da síndrome de burnout no labor do docente do ensino superior e se dirigiu exclusivamente aos docentes do Curso de Medicina, como representantes dos fatos introdutórios, que não foram descritos especificamente por eles, promovendo uma Introdução desbalanceada de variáveis de confusão. De modo geral, as revisões descreveram em grau variado de profundidade as condições de saúde de interesse, as exposições que afetam a condição de saúde de interesse e a importância em conduzir a revisão.

Tecnicamente, esperava-se que, na Introdução das revisões, fossem descritos os objetivos específicos, incluindo quaisquer hipóteses pré-existentes. Neste estudo, 33\% deles não explicitaram o objeto do estudo. As demais revisões descreveram-no utilizando linguagem simples e acessível para os profissionais familiarizados ou não com a problematização investigada.

\subsection{Métodos das revisões elencadas}

As revisões elegíveis neste estudo, exceto uma que não fez, apresentaram os critérios estruturantes da revisão na seção Materiais e Métodos. Duas revisões usaram uma figura para demonstrar o diagrama de fluxo seguido para o processo de seleção dos artigos da amostra, outros três informaram a quantidade de estudos excluídos sem especificar, e outro, nada mencionou a respeito.

Como a definição e relato das características do estudo usadas como critério de elegibilidade para inclusão e exclusão na literatura, a maioria das revisões, assim como esta, especificou um determinado período (em anos) para 
ser considerado e direcionamento do estudo. Enquanto duas revisões (33\%) restrições de idioma, outras três (50\%) só avaliaram professores universitários da área da Saúde, e nenhuma das revisões estabeleceu critérios de relevância dos artigos selecionados.

A maioria das revisões descreveu as fontes de informação na pesquisa (por exemplo, bancos de dados, mecanismos de pesquisa e periódicos selecionados). A metade dos registros utilizou como ferramenta a plataforma do Google Acadêmico, sendo que um dos registros utilizou esse recurso como única fonte de pesquisa. Essa base de dados, embora endossada por alguns, apresenta limitações, visto que alguns periódicos são destinados a associados e cobram uma taxa para acesso ao texto integral. Também, devido a critérios de indexação ao Google Acadêmico, algumas obras relevantes podem não ser encontradas apenas por não considerado essas especificações em sua produção, em alguns casos, podendo apresentar estudos de fontes desatualizadas e de pouca confiança e, no pior dos cenários, trabalhos que não sejam nem mesmo acadêmicos (SANTOS; SANTOS, 2017).

Em relação à data da pesquisa, três revisões (50\%) informaram explicitamente o mês e o ano da pesquisa. A maior parte das revisões não forneceu a lista completa de palavras-chave e termos empregados na pesquisa nas bases de dados, aspecto que não permite que outros pesquisadores repitam a pesquisa. No conjunto das revisões, somente duas relataram como os registros recuperados foram examinados (triagem de títulos e resumos). Além disso, as revisões carecem de transparência em termos de explicação dos diferentes aspectos do processo de coleta de dados, incluindo o método de gerenciamento e se os participantes dos estudos eram representativos de toda a população de onde foram recrutados.

\subsection{Resultados e discussão das revisões elencadas}

Exceto uma, as demais revisões relataram o número de estudos identificados a partir de bases de dados ou fontes eletrônicas. Não houve registro de divulgação de duplicatas e das falhas em atender aos critérios de elegibilidade. $O$ número de artigos incluídos nas análises foi divulgado em quatro destas revisões. Cinco revisões relataram a síntese dos artigos selecionados, forneceram a lista de artigos incluídos no texto e os citaram nas referências bibliográficas.

Os resultados foram resumidos em seis revisões e todas elas forneceram um resumo dos principais resultados. As limitações da revisão foram abordadas por uma única revisão. Nenhuma revisão se ateve à heterogeneidade dos resultados, sendo esperada principalmente em estudos observacionais. Assim, fica evidente a manutenção do viés de publicação, em que os trabalhos com efeito positivo aparecem em maior número que os trabalhos com efeitos negativos. Desse modo, as revisões tendem a perpetuar as informações de preferência dos periódicos primários.

Entre os diferentes tipos de análise conduzidos por estudiosos, as revisões sistemáticas se apresentam como uma robusta estratégia para avaliar 
a atual condição do conhecimento sobre o bem-estar do docente universitário. Os resultados de nossa análise não corroboram com essa tendência, pois indicam um número reduzido de revisões realizadas na última década. O nosso estudo também levanta elementos sobre os processos e procedimentos empregados pelos autores. Mais especificamente, surge a questão de saber se as revisões rotuladas como 'sistemáticas' empregam realmente diretrizes e práticas sistemáticas para revisar a literatura.

Um ponto importante a ser considerado, denotam que as revisões da literatura geralmente envolvem procedimentos e práticas que podem não ser necessariamente bem objetivos. Nesse sentido, empregar diretrizes norteadoras consagradas é importante para minimizar vieses e produzir avaliações confiáveis do corpo de conhecimentos existente.

O nosso estudo também mostra que apenas a metade das revisões seguiu algum protocolo conhecido. Além disso, na maioria das revisões elencadas, muitos dos critérios de elegibilidade não foram levados em consideração. Por exemplo, as palavras-chave específicas selecionadas para pesquisar nos bancos de dados muitas vezes não eram especificadas nas revisões publicadas, ou a contaminação das conclusões devido ao envolvimento de professores não universitários na amostra, ou a apresentação de resultados baseados em estudos estrangeiros com aplicação direta a casos brasileiros.

De modo ilustrativo, uma das revisões apresentou um estudo realizado com 437 docentes holandeses de Cursos de Medicina no ano de 2014, no qual o início da carreira (menor idade do professor) apareceu como fonte de exaustão emocional para $25 \%$ dos pesquisados (TIJDINK, VERGOUWEN; SMULDERS, 2014). Os revisores, de 'modo duvidoso', baseados em um único estudo estrangeiro, 'misturaram' aquele resultado com outros dois estudos nacionais e concluíram que estar em início de carreira seria um desencadeador da síndrome de burnout. Entretanto, as pesquisas acerca dos anos de experiência mostramse diferentes, pois a síndrome é muito mais um processo crônico que se consolida com o tempo, o que contraria a visão da variável 'idade', de forma que esta variável mostra associação inconsistente na literatura atual (TRIGO; TENG; HALLAK, 2007). Essa preocupação poderia ser ampliada ao estendê-la à seleção de estudos com amostra reduzida $(n=6)$ ou não informar o número da amostra, ao descuido com a duplicidade de estudos selecionados, ao enfoque em uma causa específica investigada (como seria um problema de voz) ou a um grupo específico de professores.

De modo curioso, em relação às investigações destinadas a grupos específicos de professores, por se tratar de saúde ocupacional, o tema, na maior parte das revisões, focou em professores universitários da área da Saúde. Criase um vínculo interessante em que pesquisadores interessados em saúde desviam o olhar para aqueles que trabalham nesta área. O perigo não está no direcionamento, o perigo está na tendência à generalização.

As inconsistências emergentes ao comparar as diferentes revisões apresentadas podem ser atribuídas à natureza multidisciplinar do tema, que abrange um campo vasto a ser pesquisado. $O$ assunto é investigado por grupos 
com diferentes formações educacionais, que envolvem saúde, trabalho, educação, sociedade, economia e ambiente. Como tal, não surpreende que diferentes considerações sejam manifestadas. Isso não facilita acordos sobre o tipo de protocolos ou listas de verificação a serem usados ao produzir uma representação do corpo de conhecimento (LIMA et al., 2012). Além disso, embora a maioria das revisões forneça implicações e recomendações para pesquisas futuras, suas conclusões raramente permitem que os pesquisadores identifiquem tendências atuais e futuras homogêneas.

\subsection{Conclusão das revisões elencadas}

Todas as revisões forneceram uma Conclusão ou Considerações Finais, sendo que a metade dos registros oferece sugestões para pesquisas futuras. Nenhuma das revisões descreveu fontes de financiamento para a realização do trabalho.

Ficou evidente que a maioria das revisões não considerou muitos dos itens que constituem o protocolo PRISMA para revisões sistemáticas. Verificaram-se várias revisões sistemáticas que não forneceram uma explicação clara de seu processo de coleta de dados, levando a uma falta de transparência dos resultados para poder responder à problematização. Um diagrama de fluxo explicando as etapas do processo sistemático de revisão não foi incluído em várias destas revisões avaliadas. Desse modo, as conclusões não responderam de modo claro o problema do estudo, ou o fizeram de modo duvidoso, talvez devido a vieses de seleção.

\subsection{Limitações do método}

Antes de considerar esses resultados e ponderá-los para, de algum modo, atender-lhes, é importante ressaltar pelo menos dois pontos. A quantidade de revisões encontrada não foi suficiente para 'encerrar o assunto'. Desse modo, é prudente considerar a possibilidade de realizar outro grupo de revisões, que apresentem padrão de qualidade diferente das elencadas. Não foi procurada a 'literatura cinzenta', aquela não indexada em periódicos que publicam formalmente a literatura científica, que consiste em aproximadamente $10 \%$ de estudos referenciados pelas revisões da Cochrane. Ressalta-se como ponto forte deste estudo a busca abrangente de trabalhos publicados e a discussão dos resultados no contexto da própria revisão sistemática e em relação à literatura prévia, na qual todos os estudos que foram selecionados tiveram sua elegibilidade confirmada pela leitura integral do artigo. Assim como na etapa de triagem, a confirmação da elegibilidade foi realizada por duplas de revisores, de modo independente.

\section{Considerações finais}

A partir de limitações das revisões sistemáticas encontradas mediante a análise do presente estudo, é sugestivo que permanece uma necessidade urgente de consistência das revisões sistemáticas nesse campo de investigação. 
A qualidade das revisões da literatura existentes sobre o acometimento de professores universitários pela síndrome de burnout mostra uma necessidade urgente de estudiosos em campo adotarem protocolos desenvolvidos para nortear as revisões sistemáticas, com destaque aos recursos para explorar a heterogeneidade dos resultados, visto que a presença de heterogeneidade é esperada em revisões da literatura, principalmente em estudos observacionais e sempre será uma limitação inerente. Ao fazer isso, futuras revisões sistemáticas podem se basear em critérios mais transparentes e confiáveis, minimizando suposições implícitas e vieses.

Este estudo visa também a orientar qualitativamente a elaboração e condução estruturada de uma revisão da literatura. No entanto, ele não considera a relevância do tipo de questão no processo da revisão e não esgota todos os mecanismos necessários para entender e realizar uma metanálise. 0 detalhamento de alguns conceitos fundamentais necessários para ampliar o entendimento das metodologias, principalmente quando propostas em diferentes áreas do conhecimento e com finalidades diversas daquela da saúde, é um desafio para trabalhos futuros.

\section{REFERÊNCIAS}

ARAÚJO, Carlos. Bibliometria: evolução histórica e questões atuais. Em Questão, Porto Alegre, v.12, p. 11-32, 2006.

CAMPBELL, Mhairi; MCKENZIE, Joanne; SOWDEN, Amanda; KATIKIREDDI, Srinivasa Vittal; BRENNAN, Sue; ELLIS, Simon; HARMANN-BOYCE, Jamie; RYAN, Rebecca; SHEPPERD, Sasha; THOMAS, James; ELCH, Vivian; THOMSONET, Hilary. Synthesis without meta-analysis (SWiM) in systematic reviews: reporting guideline. BMJ, London, 368, el6890, 2020. Disponível em: https://pubmed.ncbi.nlm.nih.gov/31948937/. Acesso em: 15 mar. 2020.

CODO, Wanderley. Educação: carinho e trabalho. Petrópolis: Vozes, 1999.

FERREIRA, Elisandra Gonçalves; SILVA, Franciele Cascaes; BENTO, Gisele Graziele; SOLEMAN HERNANDEZ, Salma Stéphany; BERNARDO, Valdeni Manoel; DA SILVA, Rudney. Revisão sistemática sobre síndrome de burnout e atividade física em professores. Educación Física y Deporte, Medelin, v. 34, p. 309-330, 2015. Disponível em:

https://pesquisa.bvsalud.org/portal/resource/pt/biblio-831036. Acesso em: 14 jan. 2020.

FERREIRA, Francielly Damaris; BORGES, Cristiane José. Síndrome de burnout: uma reflexão literária sobre a ocorrência em docentes do ensino superior.

Revista Eletrônica do Curso de Pedagogia do Campus Jataí, Jataí, v. 8, p. 1-18, 2010. 
FREITAS, Ellen de Souza, SENRA, Luciana Xavier Breve análise bibliométrica sobre a síndrome de Bornout em professores universitários. Revista

Científica da Faminas, Muriaé, v. 11, p. 119-138, 2015. Disponível em: http://200.202.212.131/index.php/RCFaminas/article/view/115. Acesso em: 12 dez. 2020.

\section{HIGGINS, Jullian; GREEN, Salien. Cochrane Handbook for Systematic}

Reviews of Interventions, Version 5.1.0. Melbourne, Australia (AUS): Department of Health and Ageing, Austrália. The Cochrane Collaboration. 2011. Disponível em: www.cochrane-handbook.org. Acesso em: 23 out. 2019.

LEITE, Andrea Ferreira; NOGUEIRA, Júlia Aparecida Devidé. Fatores condicionantes de saúde relacionados ao trabalho de professores universitários da área da saúde: uma revisão integrativa. Rev Bras Saude Ocup, São Paulo, v. 42, p. 1-15, 2017. Disponível em:

https://www.scielo.br/pdf/rbso/v42/2317-6369-rbso-42-e6.pdf. Acesso em: 21 out. 2020.

LIMA, Dartel Ferrari; MALACARNE, Vilmar; STREIEDER, Dulce Maria. O papel da escola na promoção da saúde - uma mediação necessária. Eccos - Rev. Cient., São Paulo, n. 28, p. 191-206, maio/ago. 2012. Disponível em: https://periodicos.uninove.br/eccos/article/view/3213/2322. Acesso em: 21 out. 2020.

MARTINS, Júlia Trevisan; RIBEIRO, Renata Perfeito; RIBEIRO, Benedita Gonçalves de Assis; ROBAZZI, Maria Lucia do Carmo Cruz; VEIGA Eugenia Velludo. Bem-estar de docentes universitários: revisão integrativa da literatura. Revista Varia Scientia - Ciências da Saúde, Cascavel, v. 2, p. 156-164, 2016. Dispnível em: http://erevista.unioeste.br/index.php/variasaude/article/view/14885. Acesso em: 21 nov. 2020.

MOHER, David; SHAMSEER, Larissa; CLARKE, Mike; GHERSI, Davina; LIBERATI, Alessandro; PETTICREW, Mark; SHEKELLE, Paul; STEWART Lesley; PRISMA-P Group. Preferred reporting items for systematic review and metaanalysis protocols (PRISMA-P) 2015 statement. Systematic Reviews, London, v. 4, p. 2-9, 2015. Disponível em:

https://systematicreviewsjournal.biomedcentral.com/articles/10.1186/20464053-4-1. Acesso em: 15 out. 2020.

PAGE, Matthew; SHAMSEER, Larissa; ALTMAN, Douglas; TETZLAFF, Jennifer; SAMPSON Margaret; TRICCO, Andrea; CATALÁ-LOPEZ, Ferrán; EMMA, Lun Li; REID, Emma; SARKIS-ONOFRE, Rafael; MOHER, David. Epidemiology and Reporting Characteristics of Systematic Reviews of Biomedical Research: A Cross-Sectional Study. PLoS Med, San Francisco, v. 13, p. e1002028, 2016. Disponível em: 
https://journals.plos.org/plosmedicine/article?id=10.1371/journal.pmed.10020 28. Acesso em: 20 nov. 2020.

PEREIRA, Raissa Cristina; RIBEIRO, Lucas Augusto de Carvalho; REIS, Euclides Fernandes; FERNANDES, Patricia de Souza. Síndrome de Burnout em professores de medicina: revisão sistemática. Revista EDaPECI, Aracajú, 18, 115-126, 2018. Disponível em:

https://seer.ufs.br/index.php/edapeci/article/view/10026. Acesso em: 05 dez. 2020.

SAMPAIO, Adelar Aparecido; STOBÄUS, Claus Dieter; BAEZ, Marcio Alessandro Cossio. Vivências de mal-estar na transição da licenciatura à docência.

Movimento, Porto Alegre, v. 23, p. 975-988, 2017. Disponível em: https://seer.ufrgs.br/Movimento/article/view/65257. Acesso em: 01 dez. 2020.

SAMPAIO, Adelar Aparecido; STOBAUS, Claus Dieter; LIMA, Dartel Ferrari; MAZZARDO, Oldemar; PIOVANI, Verónica Gabriela Silva; BOTH, Jorge. Validação do questionário saúde docente para o contexto Brasileiro. J. Phys. Educ. v. 32, e3228, 2021. Disponível em: https://www.periodicos.uem.br/ojs/index.php/RevEducFis/article/view/48749. Acesso em: 20 abr. 2021.

SANTOS, Maria Eduarda de Oliveira; SANTOS, Eliete Correia. O Google Acadêmico como mecanismo de auxílio na construção de trabalhos científicos e correlato ao letramento informacional. In: SEMINÁRIO DE SABERES

ARQUIVÍSTICOS (SESA): Memória, Letramento e Contribuições, João Pessoa, n. ago/2017, p. 307-320, 2017. Disponível em:

http://www.ufpb.br/evento/index.php/viii/sesa/paper/view/4594. Acesso em: 30 nov. 2020.

SOUSA, Luís Manuel Mota; FIRMINO, Cristiana Furtado; MARQUES-VIEIRA, Cristina Maria Alves; SEVERINO, Sandy Silva Pedro; PESTANA, Helena Castelão Figueira Carlos. Revisões da literatura científica: tipos, métodos e aplicações em enfermagem. RPER, Angra do Heroísmo - Portugal, v.1, p.45-54, 2018. Disponível em: https://core.ac.uk/download/pdf/232112845.pdf. Acesso em: 22 fev. 2020.

SZELIGA-KOWALCZYK, Anna. Teachers occupational burnout syndrome in secondary school. Jagiellonian Journal of Management, Warsaw, $v .1$, p. 353-367, 2015. Disponível em: https://www.ejournals.eu/jjm/Tom-1(2015)/Numer-4/art/6777/. Acesso em: 21 dez. 2020.

TIJDINK, Joeri; VERGOUWEN, Anton; SMULDERS, Yvo. Emotional exhaustion and burnout among medical professors; a Nationwide survey. BMC Medical Education, Rockville, v. 14, 183, 2014. Disponível em: https://pubmed.ncbi.nlm.nih.gov/25189761/. Acesso em: 02 nov. 2020. 
TRIGO, Telma Ramos; TENG, Chei Tung; HALLAK, Jaime Eduardo Cecílio. Síndrome de burnout ou estafa profissional e transtornos psiquiátricos. Rev. psiquiatr. Clin., São Paulo, v. 34, p. 223-233, 2007. Disponível em: https://doi.org/10.1590/S0101-60832007000500004. Acesso em: 20 mar. 2021.

VON ELM, Erik; ALTMAN, Douglas Altman; EGGR, Matthias; POCOCK, Stuart; GOTZSCHE, Peter; VANDENBROUCKE, Jan. Strengthening the Reporting of Observational Studies in Epidemiology (STROBE) statement: guidelines for reporting observational studies., Jan. BMJ, London, v. 335, p. 806-808, 2007. Disponível em: https://www.ncbi.nlm.nih.gov/pmc/articles/PMC2034723/. Acesso em: 30 jan. 2021.

WORMALD, Richard; EVANS, Jennifer. What Makes Systematic Reviews Systematic and Why are They the Highest Level of Evidence? Ophthalmic Epidemiology, Buren, v. 25, p. 27-30, 2017. Disponível em: https://www.tandfonline.com/doi/full/10.1080/09286586.2017.1337913. Acesso em: 22 mar. 2021. 